\title{
THE MATHEMATICAL BASIS OF STANDARDS ${ }^{1}$
}

\author{
Ken Krechmer \\ FELlow, InTERnATIONAL CENTER FOR STANDARDS RESEARCH
}

\begin{abstract}
Standards are fundamental to any rigorous form of communications and are used in every civilization. Given that mathematics provides a rigorous way to understand very basic phenomena, this paper explores the mathematical basis of standards. Five successions of standards are identified from pre-history to the present and mathematical models of each are presented. These mathematical models more rigorously define the standards successions proposed. The impact of these standard successions on existing standardization issues is examined.
\end{abstract}

$\mathrm{T}$ he ubiquity of technical standards in all human societies argues for a rigorous understanding - why are standards necessary? At SIIT 2001, Krechmer (2001) proposed to answer this question using set theory and information theory. The current paper builds on that paper. It develops a philosophical basis for technical standards, models the mathematical relationship between standards and entropy, and identifies five successions of standards.

The term "standard" often refers to published documents or the output of specific standardization committees. This is an application view of standards. This paper focuses on a conceptual view of standards. A standard is defined as: A codification for a society of the constraints used for one or more comparisons between implementations. This definition is taken from Krechmer, 2005 which discusses prior definitions of standards and the rational behind this definition. Standardization is the term used to refer to the application of creating, implementing or using a standard. This paper concludes by using the new conceptual models of standards to better comprehend the impact of standardization on society and technology.

\section{Developing a Rigorous View of Standards}

The idea that there should be a rigorous basis for standards is derived from a basic premise. Kant (1800) first developed the idea that a comparison is necessary for any form of understanding. Any communication requires common agreements (often termed standards). Each communication requires a comparison in the receiver between the transmitted signal and a reference. The reference is an agreement between the transmitter and the receiver; in public communications systems this reference may be a standard.

As example, in the course of reading, a word appears of unknown meaning. The reader refers to a dictionary which defines the relationship between words and their meanings. Assuming that the author also uses a similar dictionary, the reader looks up the unknown word. Upon finding the same word (a comparison), the reader now understands the meaning of the word. This three phase process, apply common reference, compare received signal to reference, and identify signal, occurs in any communications process.

For communications to occur, any transmitter and receiver must have a common reference, whether it be base pairs in DNA (the bonds between the base pairs A-T and G-C provide the reference), human language (dictionaries and formal syntax maintain the reference), ASCII characters, specific frequencies, voltages or common protocols between a transmitter and receiver. In the last four examples, the reference is maintained using published documents which may be termed standards. In all of these examples and all other communications systems, a specific relationship between the transmitter and receiver is used as a reference (by the receiver), which provides a basis for comparison, which makes communications possible.

Shannon and. Weaver (1963) offer a more technical view of this three phase process in Theorem 8, which offers the statement: “...the system of constraints [is] considered as a channel..." Figure 1 shows the relationship between a system of constraints and a communications system. Figure 1 identifies that the system of constraints includes aspects of the transmitter, the receiver, and the transmission link. Figure 1 is taken from Shannon and.

\footnotetext{
${ }^{1}$ A version of this paper titled, Modeling System Variation, was presented to International Conference on Informatics in Control, Automation and Robotics, under the topic area System Modeling, September, 2005.
} 
Weaver (1963), with the addition of the dotted "System of constraints" and a change of the word "channel" to "Transmission link."

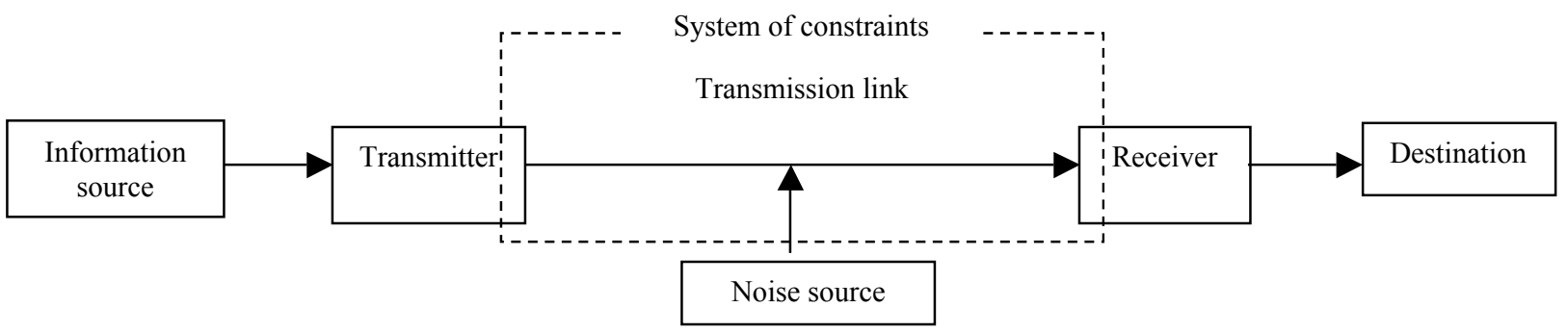

Figure 1. Communications system.

Figure 1 is the classic model of a communications system with the addition of the system of constraints. A technical standard is the reference that is required for communications. The system of constraints in this model provides the reference for a communications system. But a more complete view of the system of constraints is needed to understand the concept of standards.

\section{Model of an Information Channel}

The system of constraints includes the agreements that constrain the transmission link, transmitter and receiver (entities) and the implementation of those entities. Thus Shannon's "system of constraints" consists of multiple sets of constraints. When desired by a society, the definitions of the system of constraints become standards. Implementations of such standards create the information channel and enable communications. In this manner, standards define the bounds of the communications system. The standardized implementations of the transmission link, transmitter and receiver are then limited by these bounds.

Figure 2 presents an information channel (Abramson, 1963) consisting of a transmitter of alphabet A with elements $a_{i}$ and total elements $t$ and a receiver of alphabet $B$ with elements $b_{i}$ and total elements $r$. In order for communications to occur, the transmission link constraints are taken into account in the choice of alphabets $\mathrm{A}$ and B.

A pre-existing common set (the reference or standard) must exist between alphabet A and alphabet B prior to the elements $\left(b_{i}\right)$ of alphabet $B$ having on average a high probability relationship (communications) with the elements $\left(\mathrm{a}_{\mathrm{i}}\right)$ of alphabet A. Without such a fixed common set (during data transfer), "the comparison is between apples and oranges." This pre-existing common set may be seen as a set of constraints, S.

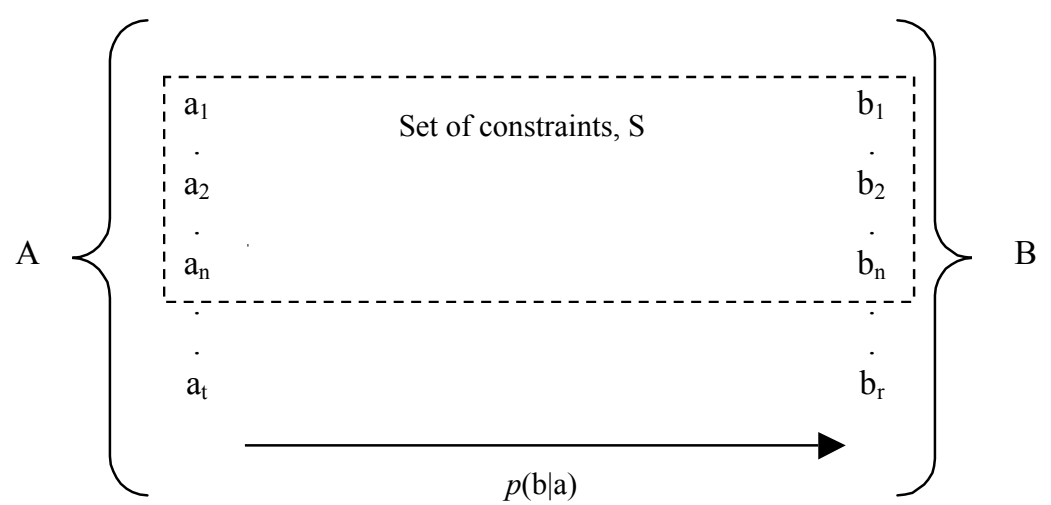

Figure 2. An information channel.

If alphabet $\mathrm{A}$ and alphabet $\mathrm{B}$ are related by the existence of a common set of constraints $\mathrm{S}$, then there is a common set of $a_{i}$ and $b_{i}$ elements numbering $n$, and $S=A \cap B$. When $S=A \cap B$ then $n \leq r$ or $t$. $S>0$ and $S=A$ $\cap B$ are the constraints that makes possible an average high probability relationship between $a_{i}$ and $b_{i}$ during communications. 
The set of constraints S exists because of a standard which may have been created by accident, assumption, convention or market (spontaneous order), or by fiat, regulation or committee (made order). Standardization is the term often used for the process of creating a standard by made order (Hayek, 1973).

An example of an information channel is a human transmitter using the 26 letter English alphabet $a_{t}$ through $z_{t}$ and a human receiver using the same alphabet $a_{r}$ through $z_{r}$. One condition for error free communications is that the humans use a common alphabet. This condition is a set of constraints consisting of the 26 pairs $a_{t}$ and $a_{r}$, $b_{t}$ and $b_{r}, \ldots . z_{t}$ and $z_{r}$. Using this set of constraints one requirement for humans communications is met. Without such a common set (before communications) no reference exists and no comparisons are possible. When the same 26 letter alphabets are used by each person, communications can occur. The entropy of specific letters or letter groups used may then be calculated.

The set of constraints shown in Figure 2 is congruent with the system of constraints shown in Figure 1. This indicates that the model in Figure 2 offers the basis for a more complete description of the bounds of a communications system.

\section{Relating Standards to Entropy}

As described above, standards define the bounds of the information channel including the transmitter and receiver. Describing the relationship between the transmitter and the receiver that enables communications first requires a description of each of the entities. Figure 2 models the transmitter and receiver as two sets $\mathrm{A}$ and $\mathrm{B}$. Using information theory the simplest possible description of the information in a single set (A) is:

$H(A)=-\sum_{i=1}^{i=n} p\left(a_{i}\right) \log p\left(a_{i}\right)$

The equation above describes the entropy $(\mathrm{H})$ of set $\mathrm{A}$. The maximum limit of $\mathrm{H}(\mathrm{A})$ is $\log \mathrm{n}$, which occurs when all the probabilities of $a_{i}$ are equal. As such, $\log n$ represents the bound of the set $A$ and $H(A)$ approaches this bound as a limit. This is a fundamental point: the logarithm of the number of members of a set (the limit of the set), plus the description of the set (in this case, set $\mathrm{X}$ with discrete random variable $\mathrm{x}_{\mathrm{i}}$ ), completely bounds the set in information theoretic terms. The combination of the set description and $\log n$ is the basis of comparison of set $A$, or a standard - if codified by a society.

The information channel shown in Figure 2 identifies the two alphabets required for communications - the transmitter and the receiver alphabets. Assuming that these alphabets define only the coding, other necessary parameters of the transmitter and receiver may include bandwidth, initialization, synchronization, training, framing, error control, link layer protocol, session layer protocol, etc. The description of these additional communications parameters entails additional sets of constraints which are supported across the information channel. The bound over all sets of constraints on a specific information channel (where $\mathrm{x}$ is the total number of sets), $\mathrm{S}_{\mathrm{x}}$ is defined as $\Sigma_{\mathrm{x}} \log \mathrm{n}_{\mathrm{x}}$. When a society agrees to make the bounds of an information channel common, a similarity standard (one standards succession) for multiple independent transmitters and a similarity standard for multiple independent receivers are defined. A compatibility standard (another standards succession) which includes members of both the transmitter and the receiver sets may also be used as the definition of the bounds of the information channel.

\section{Standards Successions}

Whitehead and Russell (1910) proposed that all of mathematics may be derived from set theory. The information theoretic model of standards developed above is expanded in this section to show that the successions of standards are also based on set theory. In Krechmer 2000, the historical context of these standards successions is identified.

A hierarchy of standards is shown in Table 1. For the remainder of this paper the term standard is used without considering whether it has been codified by a society. Table 1 identifies five successions of standards based upon the type of element, set or group of sets the standards describe. It is expected that additional standards successions beyond the five noted will be identified in the future. 


\begin{tabular}{|l|l|l|}
\hline Standards succession & Logical view & Defines \\
\hline Symbol & Element of an abstract set & Relationship \\
\hline Measurement & Element of a physical set & Physical quantity \\
\hline Similarity & Multiple sets (MS) & Entity \\
\hline Compatibility & A preexisting relationship between MSs & Relationship between entities \\
\hline Adaptability & A negotiated relationship between MSs & Negotiation between entities \\
\hline
\end{tabular}

Table 1. The successions of standards.

\section{Symbol Standards}

The first succession of standards is symbol standards. A symbol (e.g., a letter, a number, degrees of a circle, or the mathematical symbols plus [+] and minus [-]), derives its meaning only as an element in that set. As example, in the two element set of mathematical symbols, the set must be described in order to understand the symbol; a numerical digit is completely described only when its base, the description of what set the digit belongs to, is also described. Figure 3 uses Venn diagrams to describe the relationship of an element to a set. In Figure 3, each symbol $\left(\mathrm{a}_{\mathrm{i}}\right)$ or element totaling $\mathrm{n}$ in the set $\mathrm{A}$ appears as a point in $\mathrm{A}$. If set $\mathrm{A}$ has a limit (number systems do not), the limit is shown as lim A. Symbols by definition have no variation.

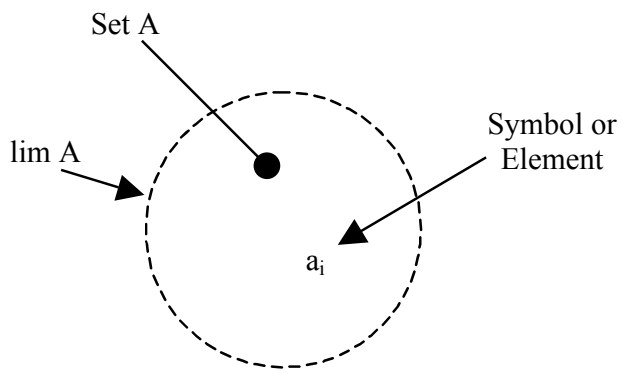

Figure 3. The relationship of an element to its set.

\section{Measurement Standards}

The next succession of standards, measurement standards (e.g., the sets of units that quantify time, space, matter and all combinations), define physical properties. Examples include the pound, meter, volt, or musical scale. The implementation used to measure or produce the units limits the size (e.g., the length of a ruler) and granularity (e.g., smallest weight on a scale) of a set of units. The smallest increment of a unit recognized by the measurement system represents an element $\left(\mathrm{a}_{\mathrm{i}}\right)$ within that measurement set. A balance scale is an implementation that quantifies weight to the smallest increment supported $\left(a_{i}=1 / n\right)$ and compares the weights of entities within the total range of increments (n) it supports. Some units (e.g., decibels) have non-linear increments, but the same concept applies. Hartley (1927) proposed that the maximum information possible in $n$ selections is bounded by $\log n$. In Figure 4, the dotted circle represents the maximum possible data $(\log n)$ of the implementation (balance scale, ruler, gauge, etc.) that is used to measure to a granularity of $1 / \mathrm{n}$ units $=\mathrm{a}_{\mathrm{i}}$. The variation among multiple measurements may be calculated statistically.

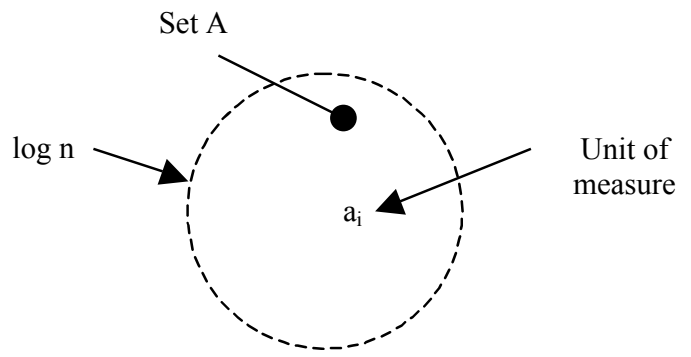

Figure 4. The relationship of a measurement implementation to a unit of measure. 


\section{Similarity Standards}

Each similarity standard is the sum of multiple sets of constraints. A similarity standard consists of one or more sets of constraints consisting of symbol standards, measurement standards and/or other similarity standards. When the number of sets of constraints is $\mathrm{x}$, the total information in a similarity standard is $\sum_{\mathrm{x}} \log \mathrm{n}_{\mathrm{x}}$. The range of an implementation of a similarity standard must be equal to or less than the maximum range allowed by the $\sum_{\mathrm{x}} \log$ $\mathrm{n}_{\mathrm{x}}$ (Figure 5). A road sign or other controlled safety sign, the mathematical signs other than plus and minus, and many common industrial products (nail, bolt, barrel, rope, brick, grade of oil, etc.) are examples of implementations of similarity standards. The specifications for a transmitter or a receiver are more complex similarity standards. The variation among implementations of a single similarity standard may be calculated statistically.

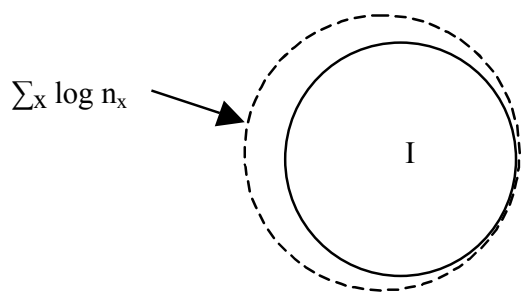

Figure 5. The relationship of the bounds $\left(\sum \mathrm{x} \log \mathrm{n}_{\mathrm{x}}\right)$ to the implementation (I).

\section{Compatibility Standards}

A compatibility standard defines the relationship between entities defined by similarity standards. A transmitter or receiver may be defined by a similarity standard which represents the bound on the range of its possible implementations. The transmitter and receiver are each described by a similarity standard independent from, but related to, the compatibility standard that defines an information channel. The set of common elements of the transmitter and receiver (set of constraints, Figure 2) is the simplest compatibility standard. The entropy (H) transferred across an information channel may be calculated as described above (Relating Standards to Entropy).

As shown in Figure 2, an information channel exists when two similarity standards have a common set S. Figure 6 offers the Venn diagram of this information channel when the number of elements of the transmitter and receiver sets are each equal to $n$. Attempting to hold $a_{t}=b_{r}=n$ is the normal practice in technical communications systems. As in Figure 2 the transmission link characteristics in Figure 6 are addressed by the choice of alphabets in the transmitter and receiver.

The Venn diagram in Figure 6 shows the case where the transmitter $(\mathrm{t})$ and receiver $(\mathrm{r})$ sets each have $\mathrm{n}$ common members. Log $a_{n}$ is the bound of the transmitted entropy $\left(H_{t}\right)$ and $\log b_{n}$ is the bound of the received entropy $\left(\mathrm{H}_{\mathrm{r}}\right)$. These bounds appear as concentric circles around the related entropy $\mathrm{H}_{\mathrm{t}}$ and $\mathrm{H}_{\mathrm{r} \text {. }}$ MI (the area within the solid line lens in Figure 6) is the mutual information transferred across the information channel between the source and the destination. Figure 6 is provided for visualization not calculation, as the shapes are idealized.

Then $\log \mathrm{n}$, the limit of $\mathrm{I}(\mathrm{A} ; \mathrm{B})$, is the bound of the information channel. I(A; B) $=\log \mathrm{n}$ can occur only when the bound of the similarity standard for the receiver $(\log n)$ and the bound for the similarity standard for the transmitter $(\log \mathrm{n})$ are overlapping and congruent. Figure 6 models how the relationship between, and bounds on, $\mathrm{H}_{t}$ and $\mathrm{H}_{\mathrm{r}}$ limit the maximum mutual information. It appears that this Venn diagram represents the simplest model of these interactions. 


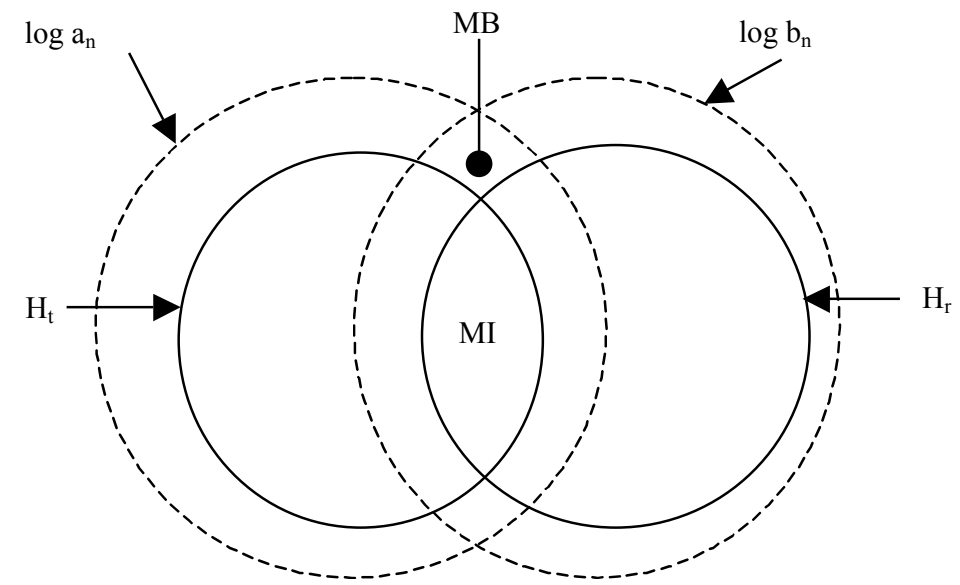

Figure 6. Venn diagram of an information channel and its bounds.

MB $\left(\log a_{n} ; \log b_{n}\right)$, the mutual bound (the lens shape enclosed in dotted lines in Figure 6), is defined as the bound of an information channel. Expanding the equation for MI above into separate joint and product entropy terms:

$$
M I=\sum_{i=1}^{i=n} p\left(a_{i}, b_{i}\right) \log p\left(a_{i}, b_{i}\right)-\sum_{i=1}^{i=n} p\left(a_{i}, b_{i}\right) \log p\left(a_{i}\right) p\left(b_{i}\right)
$$

When $p\left(a_{i}\right)=p\left(b_{i}\right)=1 / n$, the bound of set A for the transmitter $=\log n$ and the bound of set B for the receiver $=\log n$. This is the mutual bound $(M B)$ of the information channel. Inserting $1 / n$ for $p\left(a_{i}\right)$ and $p\left(b_{i}\right)$, the equation above reduces to:

$$
M B=-(\log \mathrm{n} \text { to } 2 \log \mathrm{n})+2 \log \mathrm{n}
$$

The mutual bound (MB) is some fraction of $\log n$, depending upon the ratio of the joint entropy to the product entropy when $a_{t}=b_{r}=n$. This ratio is determined by the noise in the communications system. The relationship of $a_{t}$ to $b_{r}$ to $n$, for each set of constraints in an actual implementation, is determined by the implementation of the two similarity standards (set A with $t$ elements $a_{i}$ and set $B$ with $r$ elements $b_{i}$ ) and the relationship of these similarity standards to the transmission link. When the communications system is noise free and without variation, $p\left(b_{i} \mid a_{i}\right)=1$ and $a_{t}=b_{r}=n$, then $M B=\log n$, which (along with the description of set $\mathrm{S}$ ) is the bounds of the information channel or its compatibility standard.

In specific implementations of the transmitter, receiver and transmission link, $a_{t}=b_{r}=n$ may not be true for each set of constraints in a communications system. In multiple implementations of an actual information channel such perfection is rarely attained. There may be a difference in the number of elements of the transmitter set $\left(a_{t}\right)$, or receiver set $\left(b_{r}\right)$ caused by variation in the implementation of the similarity standards. Such variation $(\mathrm{V})$, which is independent of noise, is caused by errors or misunderstandings in the related standards or their implementations. Variation $(\mathrm{V})=\left|\log b_{\mathrm{r}}-\log \mathrm{a}_{\mathrm{t}}\right|$ for each parameter set. In an efficient communications system log $\mathrm{b}_{\mathrm{r}} \geq \log \mathrm{a}_{\mathrm{t}}$. The sum of the variation of all the parameters ( $\mathrm{x}$ ) in a communications system is $\Sigma \mathrm{V}_{\mathrm{x}}$ for $\mathrm{x}=1$ to $\mathrm{x}$. As $p\left(\mathrm{~b}_{\mathrm{i}} \mid \mathrm{a}_{\mathrm{i}}\right)$ goes to 1 , MI and MB increase. As $\mathrm{V}$ goes to zero, MB goes to $\log \mathrm{r}$ as a limit, the maximum efficiency of the communications system.

The concept of $\mathrm{V}$ proposed does not include the variation allowed (by design) within a tolerance. Allowed variation, when outside the allowed tolerance, is considered a fault. Variation (V) may be caused by differences in similarity of: timer specifications, buffer sizes or revision levels (when the revisions modify the number of elements in any set in the system of constraints); and also by different options, or protocol stacks, or changes that modify the number of elements in any of these sets.

When multi-layer transmitters and receivers have a variation somewhere in the system of constraints, $\Sigma \mathrm{V}_{\mathrm{x}}$ may exist as a reduction in the maximum possible MB. As communications systems become more changeable and complex, the value of $\Sigma \mathrm{V}_{\mathrm{x}}$ is increasing due to compatibility variation. A new standards succession is emerging which offers the means to address this problem. 


\section{Adaptability Standards}

Complex communications systems utilize multiple layers of compatibility standards (e.g., protocols) each of which may exhibit compatibility variation. For application to application communications to be efficient, the sum of the total communications system variation $\left(\Sigma \mathrm{V}_{\mathrm{x}}\right)$ must be controlled, otherwise MB may be significantly reduced. The $\Sigma V_{\mathrm{x}}$ is very difficult to calculate in multi-protocol layer systems with time-independent processes, and testing all possible variations is often not practical.

To ensure that complex communications systems function properly at the applications layer, adaptability standards may be employed. Adaptability standards are a deterministic means to maximize MB. Such standards define a fully-testable independent protocol (from the data and/or control layer protocols) whose sole purpose is to negotiate among the parameters (all sets of constraints) at the transmitter and receiver to select the common sets known to fulfill the requirements necessary for a specific application-to-application communications. This capability is termed adaptability and the standards that define it are termed adaptability standards. An independent protocol, termed an etiquette (a protocol used to support adaptability), is one means to negotiate the ability to pass data or control. An etiquette only negotiates, it does not itself pass data or control information (i.e., no other layered protocol is terminated by the etiquette).

In a communications system, multiple pairs of similarity elements (used in multiple layers) exist to define a multi-layered communications interface. Changes to the similarity elements of the transmitter or receiver or their implementations may create elements that are not contained in the MB of a specific layer or reduce the MB of a different layer (e.g., by changing a buffer size which might reduce maximum packet length). Such changes are the cause of compatibility problems. When changes to the multiple compatibility standards $(\Sigma \mathrm{MB}+$, the new compatibility standards) are a superset of the previous multiple compatibility standards $(\Sigma \mathrm{MB})$, then $\Sigma$ MB remains constant or increases. However, maintaining a superset in multi-layer compatibility standards is problematic. The ability to identify a superset is made practical by requiring that an etiquette, a single tree structured protocol (which may be expanded but will always remain a superset), first negotiate between the compatibility standards and their parameters at different layers of the OSI model (X.200) to identify and select implementations that are sufficiently compatible for the desired communications application. The etiquette can perform such a negotiation based on knowledge of the desired application, existing compatibility standards or even known "bugs" using specific compatibility standards in that application.

Transmitters

Receivers

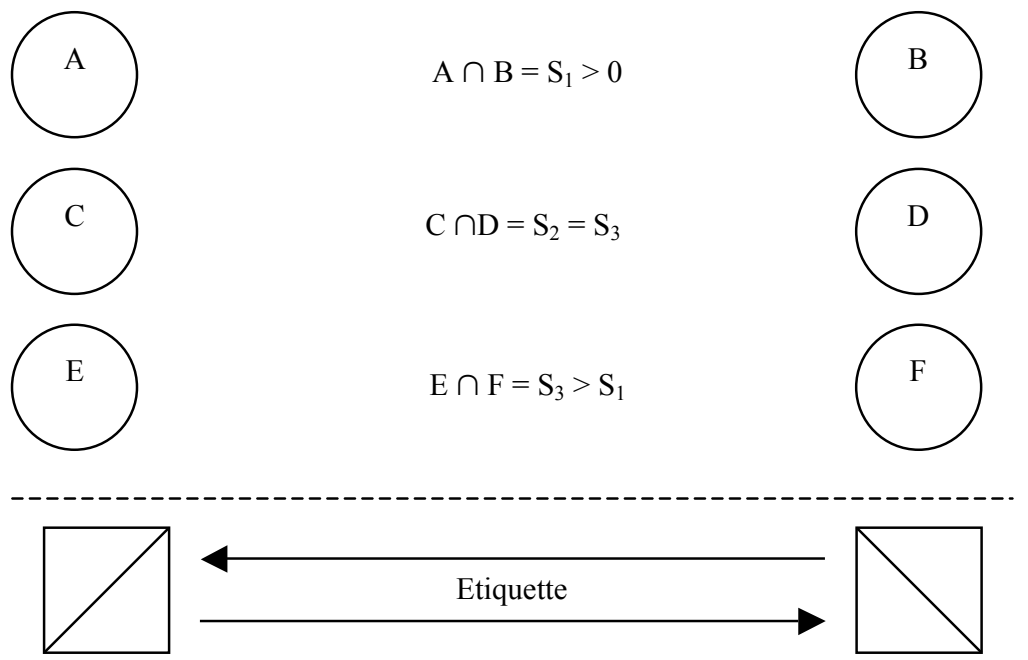

Figure 7. A multi-mode system with one etiquette

Figure 7 shows a multi-mode communications system consisting of three independent transmitter and receiver sets and one independent etiquette. Communications is possible using any one set of the compatible transmitters and receivers. The etiquette shown in Figure 7 is used to negotiate the "best" transmitter and receiver set for a specific communications application. In this example, larger $\mathrm{S}$, which offers more possible communications states, is considered better. 
In Figure 7 transmitter set $\mathrm{A}$ and receiver set $\mathrm{B}$ are compatible. Transmitter set $\mathrm{C}$ and receiver set $\mathrm{D}$ are compatible and equal (in the number of states) to transmitter set $\mathrm{E}$ and receiver set $\mathrm{F}$. Transmitter set $\mathrm{E}$ and receiver set $\mathrm{F}$ have more states than $\mathrm{A}$ and $\mathrm{B}$. In this example none of the other possible sets are compatible. In this case it is most desirable for the transmitter and receiver selected for operation to be $\mathrm{E}$ and $\mathrm{F}$ or $\mathrm{C}$ and $\mathrm{D}$. Figure 7 , without the etiquette, could also be viewed as a model of a $2 \mathrm{G}$ or $3 \mathrm{G}$ tri-mode cellular mobile and cellular base station. Figure 7, might also be viewed as a model of a multi-mode software defined radio (SDR).

There are two possible ways to select a compatible transmitter and receiver in the system shown in Figure 7. One that does not utilize the independent etiquette, called a selection mechanism and one that does, called a negotiation mechanism (etiquette).

A selection mechanism does not use the independent etiquette or any other negotiation mechanism, each transmitter transmits a signal (non-interfering) and each receiver in turn is turned on to sense the related transmit signal. The sequence of the transmitted signals determines the priority of selection of a specific transmitter and receiver in the multi-mode system. This approach has several problems:

1. Since the transmitter and receiver are not in prior communications, the two multi-mode systems (transmitters and receivers) are not initially in synchronization. This may increase the length of time needed to establish a main channel connection.

2. Given the variation of signal strength and interference in an operating environment, the three multi-mode receivers may not reliably find the compatible transmitter in all cases. Alternatively, a multi-mode receiver may identify a compatible transmitter in all cases, but this may require a significantly smaller served area per multi-mode receiver system and/or a longer time to establish the connection.

3. Considering the further complication that the multi-mode receiver does not know which of the three multi-mode transmitters may exist in any multi-mode transmitter system, it may not be practical to always select the better compatible sets $\mathrm{E} / \mathrm{F}$ or $\mathrm{C} / \mathrm{D}$ over the less desirable set $\mathrm{A} / \mathrm{B}$.

4. Since the performance of $S_{2}$ and $S_{3}$ are equal, the selection of one might be base station choice, i.e., based on the loading of the two communications systems. Alternatively, it could be mobile station choice based on the users' contractual arrangements (e.g., lowest cost service preferred). Such negotiation is not possible using a selection mechanism.

A negotiation mechanism requires an etiquette or similar mechanism to shuttle between the ends for the purpose of negotiating the "most compatible" data and control protocols. An etiquette utilizes an independent bidirectional communications channel, operating at a much lower data rate than the main channel solely for the purposes of discovery and negotiation of compatible sets. In addition to avoiding the disadvantages of a selection mechanism, the advantages of the negotiation mechanism are:

1. The low data rate of the etiquette (compared to the main channel) means that its performance (assuming the same power level as the main channel) does not reduce the coverage area or require a long synchronization time.

2. Either end system can negotiate which transmitter/receiver set to use when different compatible sets are available. In the case shown in Figure 7, the mobile and/or the base station can select for sets $\mathrm{C} / \mathrm{D}$ or $\mathrm{E} / \mathrm{F}$ as they offer equal performance.

3. Negotiation can also occur down to the revision level. Where compatibility problems have been identified in specific transmitter/receiver sets with specific revision level combinations, the most compatible sets can be negotiated. This may be a more desirable capability in SDRs as the SDR concept makes rapid change practical, with an attendant increase in compatibility issues.

4. The use of an etiquette provides a troubleshooting mechanism when a transmitter and a receiver do not establish main channel communications. When no common protocols are available for main channel communications, the differences identified by the etiquette could determine what other protocols are necessary. Future systems could even automatically access the necessary protocols (e.g., by using the Internet) to establish main channel communications.

Etiquettes are used in some existing communications systems (e.g., G3 fax T.30, telephone modems V.8, digital subscriber line transceivers G.994.1, Extended Markup Language[XML], Session Initiation Protocol [SIP]); their properties have been explored in Krechmer (2000). But the value of etiquettes is not widely understood. As example, the $3 \mathrm{G}$ cellular standards, IMT-2000, defines five different communications protocols. Currently the means of selecting a specific protocol stack is left to the designer. Existing multimode cellular handsets and base stations sense the strongest signal and give priority to higher generation protocols over lower. Such handsets and base stations support protocol selection, but cannot support protocol negotiation. For a span of time, different protocol stacks will be used in different geographic areas and the negotiation that an etiquette enables is of little use. 
Eventually however, multi-mode cellular handsets and base stations will appear; then an etiquette becomes more important, not only to negotiate around incompatibilities that emerge as more independent implementations and revisions of the communications standard exist, but also to allow the service provider to select the protocol that optimizes system loading or optimizes geographic coverage, or to allow a user to select the protocol that offers the best economic performance. The use of adaptability standards such as etiquettes is a system architecture choice that significantly enhances the long term performance of a complex communications system.

\section{Improving Communications Standards and Standardization}

The model of standards successions presented here and their logical basis appear to be a way to better understand standards and thereby address standardization issues. Communications standards may now be identified as compatibility standards (when used) and their related similarity standards. Compatibility standards are a description of the commonality between two similarity standards. Since fewer standards to describe the same entities results in less possible conflicts, creating compatibility standards (e.g., cellular air interface) as well as similarity standards (e.g., cellular mobile station and base station) is more problematic. Protocol standards which describe a transmitter, receiver and interface in a single document are the least problematic.

The difference between similarity standards and compatibility standards is subtle but significant. In order for interfaces (implementations which may be defined by compatibility standards) to be as general purpose as possible, compatibility standards should avoid (when practical) defining aspects of the transmitter or receiver related to similarity. For example, an electronic circuit compatibility standard should specify that voltages above a threshold are true, not what the voltage should be. Specifying the threshold describes compatibility. Specifying the actual voltage describes similarity.

The impact of intellectual property on compatibility standards is far more severe (on the end-user) than the impact of intellectual property on similarity standards. Controlling an attractive design or feature of a product (e.g., a clam shell cellular phone) allows the user to choose to pay for the attractive design or feature. Controlling compatibility creates a charge that all users must pay to use the communications system. Perhaps the standardization process should include a consideration of the total cost of such a ubiquitous charge when considering the value of a controlled technology in a compatibility standard.

Standardized algorithms for compression or coding (e.g., space, voice, still and moving pictures) are a form of compatibility standards as the algorithm is a common constraint in both the transmitter and the receiver. Intellectual property rights (IPR) on such algorithms are beginning to cause market fragmentation rather than market standardization. As example, the Chinese government has proposed the use of incompatible variations for DVD, cellular CDMA and 802.11 standards, possibly to avoid IPR costs.

There is a broad trend towards market led standardization in both formal and consortia standardization committees. Consortia often address IPR in compatibility standards by requiring cross-licensing. Such policies discriminate against newer market entrants, who have not developed significant IPR portfolios. Formal standardization organizations currently avoid IPR cross-licensing issues by only requiring reasonable and nondiscriminatory terms (RAND). Recognizing the negative impact on international and national standards of IPR on compatibility standards, independent of similarity standards, all standardization organizations may wish to reconsider their policies on the inclusion of IPR in compatibility standards.

Recognizing that compatibility variation should be minimized is another step toward creating "better" standards. Currently many communications standardization organizations consider ambiguity in standards acceptable and resolvable in testing. As examples, abort timers or buffers in communications systems too often have ill defined tolerance, range or resets. While testing is always desirable, it should not be used as a means to avoid more formal development procedures, as $\Sigma \mathrm{V}_{\mathrm{x}}$ in complex communications systems is beyond the current limits of testability. Methods to reduce $\Sigma \mathrm{V}_{\mathrm{x}}$ include state description languages (SDLs). A rigorous test requires that all states $(\mathrm{n})$, where $1 / \mathrm{n}$ is the smallest recognized variation of the parameter, of every parameter must be tested.

In a properly designed communications system $\log b_{r} \geq \log a_{t}$. The implementation of options in the transmitter (at any layer) increases $\Sigma \mathrm{V}_{\mathrm{x}}$ which makes the existence of such options problematic. When variability is required, etiquettes could be used to negotiate among multiple different protocol stacks rather than have a single protocol stack with options. When options exist, informative statements in the standard regarding the use and testing of the options is helpful. As example, a receiver without a specific option should be tested to ignore the option request.

A less rigorous alternative to adaptability standards or complete SDL testing would be for communications standardization committees to maintain lists by parameter type of all the individual sets of constraints in each 
compatibility standard and related similarity standards. Automated testing when revisions are proposed using such lists and/or manual review of related parameters might reduce $\Sigma \mathrm{V}_{\mathrm{x}}$.

\section{Conclusion}

This paper has shown that standards are intrinsic to any form of rigorous communications. That the secessions of standards follow a logical progression relating back to the most basic form of mathematics, set theory. Based on this basic understanding of the concept of standards communications standards have been re-examined. Communications standards have not been widely recognized as an intrinsic part of communications systems performance. This paper identifies how the long term technical performance of any communications system is strongly impacted by the standards it employs. But standards provide more than just constraints. Without them there is no communications or choice.

"...choice and constraint can coexist as partners, enabling a system, be it a living organism, a language, or a society, to follow the arrow not of entropy but of history. This is the arrow which distinguishes past from the future, by moving away from the simple, the uniform and the random, and moving toward the genuinely new, the endlessly complex products of nature and of mind" (Campbell, 1983). This is why standards are necessary.

\section{References}

Abramson, N., 1963. Information Theory and Coding, McGraw-Hill (expansion of Figure 5-8, to include the set of constraints) Campbell, J., 1983. Grammatical Man, A Touchstone Book, Simon \& Schuster, Inc., page 265.

Hartley, R. V. L., 1927. Transmission of Information, International Congress of Telegraphy and Telephony, Lake Como Italy, September 1927.

Hayek, F. A., 1973. Rules and Order, The two sources of order, page 36, Routledge \& Kegan Paul, London.

Kant, I., 1800. Logic, first published 1800 (General Doctrine of Elements, para. 6, Logical Acts of Comparison, Reflection and Abstraction), addresses the necessity of comparison for understanding.

Krechmer, K., 2000. Fundamental Nature of Standards: Technical Perspective, IEEE Communications Magazine, Vol. 38 No. 6, p. 70, June. Available at http://www.csrstds.com.

Krechmer, K., 2001. Standards, Information and Communications: a Conceptual Basis for a Mathematical Understanding of Technical Standards Proceedings of the Second IEEE Conference on Standardization and Innovation in Information Technology (SIIT 2001, Boulder, CO), page 106 - 114. Available at http://www.csrstds.com.

Krechmer, K., 2005. Definition of a Technical Standard, unpublished.

Shannon, C. E. and Weaver, W., 1963. The Mathematical Theory of Communications, page 58, Theorem 8, University of Illinois Press, Urbana and Chicago IL, USA.

Whitehead, A. N., and Russell, B, 1910. Principia Mathematica, Cambridge at the University Press. 